\title{
Rekonstruktive Forschungsmethoden rassismuskritisch perspektiviert
}

Im Bedrohungsszenario des rislamistischen Terrorismus werden Muslim:innen als Subjekte hervorgebracht. Sie haben sich zum Sicherheitsdiskurs zu positionieren und ergreifen hierzu diverse Handlungsoptionen, um auf Anforderungen und Anrufungen zu reagieren, (nicht oder anders) $\mathrm{zu}$ antworten und eigene Themen und Bedürfnisse einzubringen. Mit Foucault gehen wir davon aus, dass das gesellschaftliche Kräfteverhältnis keines zwischen aktiv Herrschenden und passiv Beherrschten ist: Macht wird nicht von einer Gruppe besessen, während andere gänzlich ohnmächtig sind. Vielmehr wirkt Macht in sozialen Beziehungen und stellt »eine bestimme Form augenblickhafter und beständig wiederholter Zusammenstöße « ${ }^{1}$ dar. Mit der vorliegenden empirischen Studie versuchen wir, jenen komplexen Prozessen Rechnung zu tragen, in denen Muslim:innen als soziale Größe produziert werden und in denen sie sich zugleich selbst hervorbringen. Es bieten sich rekonstruktive Forschungsmethoden und insbesondere die Dokumentarische Methode an, um Verhandlungen zwischen $>$ Muslim:innen $<u$ untersuchen, während die Objektive Hermeneutik sich für das Herausarbeiten der Positionierungen einzelner `Muslim:innen als nützlich erweist. Beide Methoden unterscheiden sich in ihrem Erkenntnisinteresse und -ziel: Während mithilfe der Dokumentarischen Methode der Umgang mit der bestehenden, gesellschaftlichen Wirklichkeit zu erklären versucht wird, 
zielt die Objektive Hermeneutik darauf, die Sinnstrukturierung dieser Wirklichkeit zu rekonstruieren und zu verstehen.

Im Rahmen unserer Auseinandersetzungen mit beiden Methoden wurden wir auf verschiedene Probleme aufmerksam, die mit erkenntnistheoretischen und forschungsethischen Fragestellungen zusammenhängen. Dies zog Konsequenzen für den Erhebungs- und Auswertungsprozess unserer Daten nach sich: Wir konnten in den Methodologien beider Methoden keine systematische Berücksichtigung gesellschafts- oder gar rassismuskritischer Ansätze erkennen, die es ermöglicht hätten, die Komplexität und Widersprüchlichkeit der Erfahrungen und die Gleichzeitigkeit verschiedener, auch widerstreitender sozialer Wirklichkeiten von rassifizierten Menschen deutend $\mathrm{zu}$ verstehen. Das geteilte, reflektierte Erfahrungswissen rassifizierter Personen wird im sogenannten »Regelwissen ${ }^{2}$ der Objektiven Hermeneutik oder im »kommunikative[n] Wissen $\aleph^{3}$ der Dokumentarischen Methode nicht als common sense der Gesamtgesellschaft abgebildet. Rassifizierte Personen greifen aber sowohl auf ein gesamtgesellschaftliches Regel- bzw. kommunikatives Wissen als auch auf ein Communitybasiertes zurück, das ergab unsere Analyse. Um also einen reflektierten Zugang zu Erfahrungen, Orientierungen und Handlungspraxen $\mathrm{zu}$ ermöglichen bzw. nicht durch eigene, unbearbeitete Verstrickungen $\mathrm{zu}$ behindern, sollte der Forschungspraxis zur Interpretation und Analyse von Datenmaterial mit rassifizierten Menschen eine macht- und rassismuskritische Auseinandersetzung vorangehen. Die Entwicklung der Fragestellung und des Forschungsdesigns, der Zugang zum Feld, die Erarbeitung von Diskussionsimpulsen und Leitfragen, die Vorbereitung auf die Datenerhebung und der Prozess der Interpretation und Generalisierung müssen rassismuskritisch reflektiert werden, um der rassistischen Strukturierung der gesellschaftlichen Wirklichkeit Rechnung tragen zu können.

Bohnsack, Przyborski und Schäffer argumentieren, dass die Analyse mithilfe der Dokumentarischen Methode stets im Zusammenhang mit

2 Wernet 2009: 14.

3 Bohnsack 2018: 54. 
»unserer eigenen Praxis des Forschens $\aleph^{4}$ steht. Wernet beschreibt für die Objektive Hermeneutik, dass Interpret:innen mit den Regeln sozialer Handlungen vertraut sind, da sie als »sprachlich-handlungsfähige Subjekte« gelten, sodass sie »keine Vertrautheit mit dem Forschungsgegenstand $\aleph^{5}$ aufweisen müssen. In Abgrenzung dazu stellen wir im Folgenden unsere Forschungspraxis und modifizierte Anwendung der genannten rekonstruktiven Forschungsmethoden mit (als) Muslim:innen (Markierten) vor dem Hintergrund des antimuslimischen Rassismus vor.

\section{Sample, Interviewführung und das Problem mit den Typologien}

Bezüglich der Dokumentarischen Methode orientierten wir uns an den Vorlagen für Gruppendiskussionsverfahren ${ }^{6}$ und luden (als) Muslim:innen (Markierte) zu drei Gruppendiskussionen ein, um vor dem Hintergrund ihrer beruflichen, ehrenamtlichen, aktivistischen, künstlerischen oder wissenschaftlichen Tätigkeit und ihrer Erfahrungen mit dem antimuslimischen Rassismus zu sprechen. Wir baten sie, miteinander darüber ins Gespräch zu kommen, wie sie den Sicherheitsdiskurs wahrnehmen, welche Bedeutung er für sie hat und wie sie damit umgehen. Entsprechend der Methodologie der Objektiven Hermeneutik führten wir anschließend zehn "gesprächsförmige « ${ }^{7}$ Einzelinterviews mit (als) Muslim:innen (Markierten) zur gleichen Fragestellung. Vor der Datenerhebung entwickelten wir ein Datenschutzkonzept für den gesamten Forschungsprozess. Zur Erhebung bereiteten wir offene Diskussionsimpulse bzw. Leitfragen zur Orientierung vor. Für die Interpretationen wählten wir jeweils drei Sequenzen aus, transkribierten und interpretierten sie mit dem Ziel, den Orientierungsrahmen unserer Diskussionsteilnehmer:innen und die Handlungspraxis unserer

4 Bohnsack/Przyborski/Schäffer 2010: 13.

5 Wernet 2009: 14.

6 Bohnsack/Przyborski/Schäffer 2010.

7 Wernet 2009: 58. 
Gesprächspartner:innen sowie die Strukturen, innerhalb derer sie sich bewegen, zu rekonstruieren.

Die Gruppenzusammensetzung erfolgte nach thematischen Gesichtspunkten, wobei wir uns bemühten, sie entlang unterschiedlicher Aspekte intersektional zu pluralisieren. Das weicht von der üblichen Vorgehensweise $a b$, Gruppen als shomogene, künstliche oder sheterogene, natürliche oder einer Kombination aus beiden Elementen ${ }^{8}$ zusammen zu setzen und danach $\mathrm{zu}$ ordnen, wo »sich in ihnen ein Kontrast in den Orientierungsrahmen, aber auch in Bezug auf die Erlebnisschichtung finden " 9 lässt. Grund hierfür ist, dass es nicht unser Anliegen ist, eine vergleichende Erkenntnishaltung einzunehmen und die Besonderheit eines Falles vor dem Hintergrund anderer Fälle $\mathrm{zu}$ vergleichen, sondern möglichst differenziert Deutungen und Strategien im Umgang mit ähnlichen Erfahrungen zu rekonstruieren. Machtkritische und intersektionale Theorien $\mathrm{zu}$ Rassismus (und $\mathrm{zu}$ anderen gesellschaftlichen Machtverhältnissen) weisen überzeugend darauf hin, dass die Festlegung von Personen auf Kategorien und die Suche nach stabilen Identitäten den fluiden, widersprüchlichen und komplexen Verstrickungen in gesellschaftliche Machtverhältnisse zuwiderläuft. Obwohl rassistische Topoi und ihre Effekte klar beschrieben werden können, wirken sie zusammen mit anderen Machtverhältnissen und werden von Subjekten in jeweils konkreten Situationen ausgehandelt. Insofern würde die Identifizierung von Fällen hinter den Forschungsstand $\mathrm{zu}$ (antimuslimischem und intersektionalem) Rassismus und zur diskursiven Hervorbringung des >muslimischen Subjekts` zurückfallen - und nicht substantiell zur Beantwortung unserer Forschungsfrage beitragen. Wir nehmen daher die Interrelation von Religion, Kultur, Herkunft, Klasse, Alter, Ability, Geschlecht und Sexualität in den Blick, ohne sie als Personenmerkmale $\mathrm{zu}$ verstehen. Vielmehr verstehen wir sie als vielfach verwobene und sich wechselseitig bedingende gesellschaftliche Verhältnisse, die jeweils konkrete Effekte in konkreten Situationen haben und auf die

8 Lamnek/Krell 2016: 410.

9 Amling 2015: 99. 
in changierenden und widersprüchlichen Weisen Bezug genommen wird. Aus diesem Grund erheben wir auch keine Sozialdaten, sondern arbeiten die Bezugnahmen der Gesprächspartner:innen auf Sozialdaten, Personenmerkmale und gesellschaftliche Machtverhältnisse in ihrer Bedeutung für den jeweiligen Kontext heraus.

Anstatt also essentialistische Größen als Ausgangspunkt zu setzen und soziale Machtverhältnisse in Personenkategorien zu überführen und zu festigen, gehen wir von Dynamiken aus und richten unsere Aufmerksamkeit auf jene sozialen Verhältnisse, in denen sich Macht manifestiert. Wir stellen die realen Folgen der gesellschaftlichen Konstruktion von Kategorien in Rechnung, indem wir sie in der Rekonstruktion konkreter Sequenzen als existierende Verhältnisse berücksichtigen, arbeiten jedoch im kollektiven Forschungsprozess daran, sie nicht zu reproduzieren. Wir fragen nach den Ordnungen und Strukturen als Rahmenbedingungen, die es ermöglichen, dass der Diskurs und die Dinge, die er ordnet, sich gerade auf diese Weise (und keine andere) gestalten.

Argumentationen und Strategien zum Umgang mit der Adressierung von >Muslim:innen als Sicherheitsrisiko variieren, sie changieren selbst in den Argumentationen ein und derselben Person im Laufe des Interviews oder der Diskussion. Rekonstruktionen, die darauf zielen, sozio- und sinngenetische Typen zu generieren ${ }^{10}$, würden dies als widersprüchlich und inkonsistent deuten und zum Anlass nehmen, neue Typen zu bilden. Unser Erkenntnisinteresse bezieht sich aber nicht darauf, Typen von Muslim:innen herauszuarbeiten, sie nach binären Kategorien wie Geschlecht oder Nationalität einzuteilen, miteinander zu vergleichen und mit oppositionellen (Kontroll-)Gruppen zu kontrastieren. Es interessiert uns auch nicht, ein sogenanntes >Milieu< unter dem Aspekt von Generation oder Geschlecht zu (re-)konstruieren und auf dieser Grundlage Erfahrungen oder Orientierungen zu kategorisieren. ${ }^{11}$ Mit Ordnungen und Kategorien zu arbeiten, affirmiert diese zumindest vorläufig und schließt Uneindeutigkeit aus. Bestenfalls 
werden Essentialisierungen, Homogenisierungen und Dichotomisierungen durch feinere oder neue ersetzt, nicht aber verflüssigt. Genau so funktionieren Rassismen, wie Hall eindrücklich argumentiert. ${ }^{12} \mathrm{Um}$ keine Rassismen und anderen gesellschaftlichen Machtverhältnisse zu reproduzieren, aber auch, um für die Pluralität und Ambiguität von Deutungs- und Handlungsmöglichkeiten offen $\mathrm{zu}$ bleiben, beziehen wir also Geschlecht, Alter und all die anderen machtförmigen Anrufungen in die Analyse ein und behandeln sie als gesellschaftliche Positionierungen, die sich materialisieren und bedeutsam werden können.

Für unser Forschungsprojekt sehen wir uns also vor der Herausforderung, einerseits der erneuten Rassifizierung von >Muslim:innen<, auch durch die Konstruktion neuer oder von Unter-Kategorien von >Muslim:innen $\mathrm{zu}$ widerstehen, andererseits aber die Deutungen und Handlungen im Umgang mit der rassifizierenden Adressierung als >Muslim:innen< herauszuarbeiten. Uns interessieren ganz konkret die Antworten auf die Anrufung. Insofern müssen wir die Anrufung >Muslim:in dem Sample zugrunde legen, ohne aber den Fehler zu begehen, die befragten Personen als Personenkategorie zu bestätigen: Eine Untersuchung zu Lehrkräften interviewt Lehrkräfte, eine Untersuchung zu Muslim:innen entsprechend Muslim:innen. Im Unterschied zum häufig vorgeschlagenen Vorgehen bei rekonstruktiven Forschungsmethoden entwickeln wir aber keine Subjekt- oder Gruppen-Kategorien und auch keine Binnen-Identitäten (Muslim:in-Typ a/b/c, muslimische Frauen der zweiten Generation). Vielmehr arbeiten wir Strategien heraus, die unter anderem mit der Adressierung als >muslimische Frau oder >türkischer Arbeiter zusammenhängen können, ohne sie als Typ darauf festzulegen und andere Aussagen vor diesem Hintergrund zu deuten bzw. andere Personen in diese Kategorien einzuordnen. Die Berücksichtigung ihrer Adressierung oder Selbstpositionierung dient in der konkreten Sequenz dazu, ihre Deutung bzw. ihre Strategie zu verstehen und bezieht jeweils jene Positionen, Kategorien, Machtverhältnisse usw. ein, die explizit oder implizit (an-)gedeutet werden. 
Unzusammenhängende oder widersprüchliche Aussagen einer Person oder eines >Typus` müssen also nicht als solche problematisiert und in feinere oder neue Kategorien überführt werden, um den Widerspruch aufzulösen und konsistente Typen zu generieren. Vielmehr ergeben sie unserer Vorgehensweise nach in einem spezifischen Kontext Sinn, etwa in der Konstellation der Diskussionsteilnehmer:innen, bezüglich des thematischen Fokus, wegen des intersektionalen Changierens, des Abwägens von Folgen und aus anderen Gründen, die es zu rekonstruieren gilt: $\mathrm{um} \mathrm{zu}$ verstehen, nicht um $\mathrm{zu}$ ordnen. Sie gehen also in die Interpretation der Argumentation ein, um die Deutungs- und Handlungsstrategien zu entschlüsseln und nicht, um einen Deutungsoder Handlungstyp zu (re-)konstruieren.

Wir sprechen unsere Gesprächsteilnehmer:innen also nicht analog zum hegemonialen Diskurs als >Muslim:innen monialen Unterscheidungen nach Alter, Geschlecht usw. an, sondern lassen offen, ob und gegebenenfalls in welcher Weise sie sich selbst oder auch die anderen Diskussionsteilnehmer:innen als Muslim:innen einordnen, und ob und in welcher Weise sie sich in den durch gesellschaftliche Machtverhältnisse hervorgebrachten Personenkategorien wiederfinden und darin positionieren. Unsere Fragestellung macht zudem deutlich, dass wir um die Markierung als sgefährliche Muslim:in wissen und diese Figur in der Forschungsfrage problematisieren (»Sicherheitsdiskurs«). Wir legen unser Erkenntnisinteresse offen und bitten unsere Gesprächspartner:innen, über ihre Sichtweisen zu sprechen (»wie nimmst du das wahr, wie siehst du das«), zu assoziieren und zu diskutieren, was sie an der Frage für wichtig halten. Durch die gezielte (anstelle der beliebigen oder natürlichen) Zusammensetzung der Gruppen versuchen wir theoriegeleitet, den im antimuslimischen Rassismus und insbesondere im Sicherheitsdiskurs angerufenen Positionen Rechnung zu tragen, sodass in den Diskussionen sowohl die Ambiguität als auch die Konkretheit sowie die Suchbewegungen zum Vorschein kommen. Das heißt, unser Forschungsdesign zielt nicht darauf, zur Ordnung der Dinge beizutragen, sondern im Gegenteil die Ordnung kritisch zu reflektieren, ihr Gefüge aufzubrechen, die Komplexität zum Vorschein kommen zu lassen und das zur unhinter- 
fragten Normalität Gewordene in Frage zu stellen. Insofern liegt der Fokus unserer Rekonstruktion des geteilten, aber auch differenten, kollektiven Wissens auf den ihnen zugrunde liegenden geteilten und differenten, kollektiven und spezifischen Erfahrungen, die durchzogen sind von interrelationalen, intersektionalen, gesellschaftlichen Machtverhältnissen, deren Relevanzen in der Auswertung zu berücksichtigen sind, um spezifische Deutungen und Strategien nachzuvollziehen.

\section{Nutzen und Grenzen rekonstruktiver Methoden}

Wir haben uns für die Gruppendiskussion als Erhebungsmethode und die Dokumentarische Methode als Auswertungsmethode entschieden, um einen Zugang zu kollektiven (impliziten und handlungsleitenden) Wissensbeständen von muslimischen Akteur:innen $\mathrm{zu}$ erhalten. Die Auswertung von Einzelinterviews mithilfe der Objektiven Hermeneutik hingegen dient der Rekonstruktion der diskursiven Struktur des Sicherheitsdiskurses, des Erfahrungswissens und der Handlungspraxis unserer Gesprächspartner:innen sowie ihren Subjektivierungsweisen und Selbsttechnologien im Umgang damit.

\section{Dokumentarische Methode: Kommunikatives, atheoretisches und situiertes Wissen}

Die Dokumentarische Methode eignet sich dazu, die Aushandlung von Deutungen und Umgangsweisen mit dem Sicherheitsdiskurs unter Muslim:innen zu untersuchen. In Gruppendiskussionen kann das kommunikative Miteinander mehrerer Personen und die Herstellungspraxis ihrer Wirklichkeit rekonstruiert werden. ${ }^{13}$ Laut Bohnsack, Przyborski und Schäffer bietet diese Methode die Möglichkeit, einen »Zugang zu kollektiven [H.i.O.] Erfahrungen und Orientierungen ${ }^{14} \mathrm{zu}$ erhalten.

\footnotetext{
13 Nohl et al. 2013: 21ff.

14 Bohnsack/Przyborski/Schäffer 2010: 13.
} 
Die in der vorliegenden Publikation vorgestellten Ergebnisse verweisen auf unterschiedliche Umgangsweisen mit dem Sicherheitsdiskurs durch muslimische und muslimisierte Akteur:innen. Konjunktive Erfahrungsräume sind gemeinsame Erfahrungen und Handlungspraxen (auch kollektive Deutungsmuster genannt), die Menschen zu einem spezifischen Thema teilen, ohne sich untereinander kennen $\mathrm{zu}$ müssen. Diese können an jenen Stellen der Gruppendiskussion erkannt werden, »wo diejenigen, die ein gemeinsames Problem oder allgemeiner: einen gemeinsamen [H.i.O.] Erlebnishintergrund oder existentiellen Hintergrund teilen, in ein darauf gerichtetes oder darauf aufbauendes kommunikatives Miteinander [H.i.O.] eintreten ${ }^{15}{ }^{15}$ Auch unsere Rekonstruktion zielt darauf, konkrete Handlungsstrategien herauszuarbeiten.

In Anlehnung an die Dokumentarische Methode fassten wir zunächst den thematischen Verlauf zusammen, wählten die für unsere Fragestellung relevanten Sequenzen aus, die sich durch eine besondere Gesprächsdichte auszeichnen, transkribierten sie und arbeiteten Oberund Unterthemen heraus. Daraufhin schrieben wir zu jeder Sequenz formulierende Interpretationen, auf deren Grundlage wir Diskursmodi identifizierten: antithetisch, parallel, oppositionell, univok und divergent. ${ }^{16}$ Die folgende Textsortentrennung dient dazu, das atheoretische vom kommunikativen Wissen zu unterscheiden. Beim kommunikativen Wissen handelt es sich um alltägliches Wissen. Es tritt in Schilderungen von Handlungen in Erscheinung und wird in Argumentationen und Bewertungen explizit geäußert (weil-Motive ${ }^{17}$ ). Dagegen wird das atheoretische Wissen in Erzählungen und Beschreibungen deutlich, es stellt »ein implizites oder still-schweigendes, ein konjunktives Wissen $\aleph^{18}$ dar. Es ist den Personen reflexiv nicht verfügbar und wird von den Forscher:innen rekonstruiert. Das Spannungsverhältnis zwischen den beiden Wissensformen spielt für die reflektierende Interpretation 
eine wichtige Rolle, um die Art und Weise herauszuarbeiten, wie die Themen bearbeitet werden.

Die gängige Unterscheidung beider Wissensformen kann jedoch aus unserer Sicht die unterschiedlichen Bezüge auf Wissensbestände aus einer rassifizierten oder unmarkierten Position nicht hinreichend berücksichtigen. Der Methodologie der Dokumentarischen Methode zufolge nimmt das kommunikative Wissen das öffentliche Wissen in den Blick und wird expliziert, während das atheoretische Wissen auf den Umgang damit verweist und implizit ist. Die Zuordnung zu dem einen oder anderen Wissen wird durch eine Textsortentrennung (s.o.) identifiziert. Diese Trennung vermag es aber im Zusammenhang mit Redebeiträgen von rassifizierten Personen nicht, ihre spezifische Situation zu berücksichtigen: (Als) Muslim:innen (Markierte) verfügen zwar über ein gemeinsames öffentliches Wissen, das sie mit Angehörigen des Rests der Gesellschaft teilen, jedoch stellt das »situierte Wissen « ${ }^{19}$ ebenfalls ein gemeinsames Wissen gleicher Struktur dar und muss mit berücksichtigt werden. Das bedeutet im Umkehrschluss, dass nicht nur marginalisierte Personen auf ein situiertes Wissen zurückgreifen, sondern dass auch das hegemoniale Wissen situiert ist, obwohl es sich als allgemein gültig gibt. Während die hegemonialen Wissensbestände bekannt sind, finden die Wissensbestände marginalisierter Personen kaum oder gar keinen Eingang in das sogenannte Alltagswissen, obwohl es ihnen selbst neben dem hegemonialen Wissen ebenfalls als

19 Donna Haraway spricht von situiertem Wissen und kritisiert, dass die westliche Kultur Objektivität und Wissenschaftlichkeit für sich beansprucht und hierdurch weiße Herrschaftsverhältnisse reproduziert, um sich zu legitimieren. Durch essentialistische und unkritische Argumentationsweisen schließt sie marginale Wissensbestände aus. Demgegenüber eröffnet eine Haltung, die die Situiertheit des Wissens, seine Verortung anerkennt, »die Perspektive solcher Blickwinkel anzustreben, die niemals im voraus bekannt sein können und die etwas sehr Ungewöhnliches versprechen, nämlich ein Wissen, das die Konstruktion von Welten ermöglicht, die in geringerem Maße durch Achsen der Herrschaft organisiert sind (Haraway 1995: 85). 
kommunikatives Wissen zur Verfügung steht. ${ }^{20}$ Dies wirkt sich auf die Rekonstruktionen dahingehend aus, dass wir berücksichtigen müssen, dass unsere Diskussionsteilnehmer:innen das gemeinsame und öffentliche Wissen kennen und sich darauf beziehen und gleichzeitig dieses von ihrem eigenen situierten Wissen differenzieren können. In der Artikulation des situierten, alltäglichen, kommunikativen Wissens der nicht-muslimischen Gesellschaft wiesen unsere Diskussionsteilnehmer:innen mitunter auf hegemoniale Wissensbestände hin, die sie als muslimisch Markierte homogenisieren und als Sicherheitsgefahr konstruieren. Die hegemonialen Wissensbestände werden von unseren Diskussionsteilnehmer:innen meist zitierend vorgetragen, explizit oder implizit reflektiert und der Kritik unterzogen. Gleichzeitig wird den hegemonialen Wissensbeständen anderes Wissen gegenüber gestellt, das ihre Perspektiven sichtbar macht und ihre Lebensrealitäten widerspiegelt. Wir identifizieren es daher als kommunikativ-situiertes Wissen von (als) Muslim:innen (Markierten) und bezeichnen es als Erfahrungswissen oder situiertes Wissen. Dieses Wissen wird von der nicht-muslimischen Gesellschaft nicht geteilt und ist ihr häufig auch gar nicht bekannt. Das kommunikativ-situierte Wissen von Muslim:innen ist kein allgemeines, alltägliches und kann nicht als Common-Sense der Gesamtgesellschaft vorausgesetzt werden, obwohl es als solches zur Verfügung steht. Dörner et al. weisen darauf hin, dass durch einen "Common-Sense-Blick « ${ }^{21}$ bestimmte Phänomene unberücksichtigt bleiben. Das kommunikativ-situierte Wissen unserer Diskussionsteilnehmer:innen ist als Erfahrungswissen von Alltagstheorien nicht gedeckt, sodass darauf in der Forschungspraxis ohne die von uns hier beschriebenen Modifikationen im methodischen Vorgehen nicht zurückgegriffen werden kann.

20 Indigene, Schwarze und Forscher:innen of Color haben auf ein solches Wissen hingewiesen (Denzin/Lincoln/Smith 2008; Collins 1993; Lewis 1996) und verdeutlicht, dass z.B. marginalisierte Erfahrungen und Wissensbestände sehr wohl bestehen, aber de-legitimiert werden und damit noch keinen Eingang in den eurozentrischen Wissenskanon gefunden haben. 


\section{Objektive Hermeneutik: Objektive Bedeutungsmuster und latente Sinnstrukturen}

Anknüpfend an die Rekonstruktionsergebnisse der Dokumentarischen Methode haben wir ausgewählte Personen zu unseren Einzelinterviews eingeladen, um unser Verständnis der identifizierten Umgangsweisen mit dem Sicherheitsdiskurs zu vertiefen. Zirkulär vorgehend haben wir hierfür nach und nach Personen eingeladen und zunächst dieselbe Einstiegsfrage gestellt, jedoch die Nachfragen an den Personen und ihren beruflichen und/oder aktivistischen Erfahrungen orientiert.

Die Objektive Hermeneutik eignet sich zur Rekonstruktion der unbewussten bzw. latenten Handlungsmöglichkeiten und ihrer zugrunde liegenden Bedeutungen, auf die (als) Muslim:innen (Markierte) im Umgang mit dem Bedrohungsszenario zurückgreifen. Im Zuge der Anwendung der Methode stellten wir allerdings fest, dass sich die transkribierten Interviews als »Protokolle [H.i.O.] der Wirklichkeit ${ }^{22}$ nicht ausschließlich »entlang geltender Regeln $\ll^{23}$ von allen Interpret:innen verstehen lassen. Vor dem Hintergrund unseres Forschungsvorhabens und rassismuskritischer Theorien gehen wir davon aus, dass Gesellschaften von rassistischen Strukturen durchzogen sind und jedes Mitglied einer Gesellschaft darin verstrickt ist, sodass sich sowohl weiße als auch rassifizierte Personen Strategien zum Umgang damit aneignen. ${ }^{24}$ Die unterschiedlichen Weisen des Verstricktseins in rassistische Machtverhältnisse bringt es mit sich, dass nicht angenommen werden kann, dass das Wissen zu Rassismus ein Regelwissen darstellt und die Interpret:innen zwangsläufig darüber verfügen, sodass sogenannte übersubjektive Strukturen bzw. Latenzen herausgearbeitet werden können.

In Anlehnung an sinnverstehende Wissenschaften geht die Objektive Hermeneutik davon aus, »dass sich die sinnstrukturierte Welt durch Sprache konstituiert und in Texten materialisiert «. ${ }^{25}$ Die Welt

\footnotetext{
22 Wernet 2009: 12.

23 Ebd.: 18.

24 Mecheril 1994: 57ff.

25 Wernet 2009: 11.
} 
ist demnach durch Regeln vorstrukturiert und das soziale Handeln orientiert sich an den bestehenden Regeln. Für die Interpretation ist die Berücksichtigung und Einbeziehung dieser Regeln relevant, damit die Rekonstruktion sozialer Praxis gelingt. Im Mittelpunkt der Interpretation stehen also die Alltagshandlungen der Gesprächspartner:innen, die dahingehend analysiert werden, welche unbewussten bzw. latenten Regeln und Strukturen ihrem Handeln zugrunde liegen. Diese Regeln und Strukturen sind objektiv vorhanden und universal, sodass »die fundamentale Bedeutung der Regelgeleitetheit [...] in ihrer Nicht-hintergehbarkeit [besteht]. Die Lebenspraxis kann sich ihr weder entziehen, noch kann sie die Regelgeltung außer Kraft setzen ${ }^{26}{ }^{26}$ Wenn aber die Welt aus viel komplexeren, widersprüchlicheren und vielschichtigeren Regeln besteht, wie dies etwa durch unterschiedliche Positionen, Normalitäten, Regeln und Ressourcen aufgrund von Rassismus und anderen Machtverhältnissen der Fall ist, so stellt sich die Frage, ob bzw. wie sie mit dieser Methode eingefangen werden können. Die Erfahrungen von (als) Muslim:innen (Markierten) stellen eine spezifische gesellschaftliche Wirklichkeit dar, die sich unter anderem durch die Erfahrung von (intersektionalem antimuslimischem) Rassismus von der sozialen Wirklichkeit anderer Gesellschaftsmitglieder unterscheidet und nicht mithilfe sogenannter universaler Regeln rekonstruiert werden kann. Vielmehr bedarf es zur Anwendung von Regeln eines fundierten Wissens über die Funktionsweisen von Rassismus, um regelgeleitet vorgehen $\mathrm{zu}$ können. Andernfalls gehen die unreflektierten, rassistischen Wissensbestände als latente Sinnstrukturen leitend in die Interpretation ein, sodass (als) Muslim:innen (Markierte) und ihre Alltagshandlungen als anders, fremd und bedrohlich affirmiert werden. Sich an einer sogenannten universalen Sprache für den deutschsprachigen Kontext zu orientieren, bedeutet also auch, die Interpretationen entlang der hegemonialen Konstruktion von >Muslim:innen vorzunehmen. Für die Rekonstruktion ihrer Handlungspraxis erscheint es demgegenüber relevant, ihre 
Rassismuserfahrungen und die im hegemonialen Wissen ausgeblendeten Lebensrealitäten mitzudenken und generell im Regelwissen das Mitlaufen von Rassismus, also Rassismus als Subtext, Kontext oder Haupttext, mit einzubeziehen.

Eine solche Interpretation ermöglicht es, die Auswahl der Handlungsmöglichkeiten (also die Selektivität des Handelns) von (als) Muslim:innen (Markierten) vor dem Hintergrund eines rassistischen Regelwissens $\mathrm{zu}$ interpretieren und $\mathrm{zu}$ rekonstruieren. Leanza schlägt vor, die rekonstruierten Fallstrukturen der Kritik zugänglich zu machen und auch die sichtbar gemachten latenten Sinnstrukturen »als Selektion aus einem Raum von Möglichkeiten « $\mathrm{zu}$ verstehen, sodass »bestimmte Implikate von Deutungen oder aber auch versteckte Herrschafts- und >Entfremdungsstrukturen « aufgedeckt werden ${ }^{27}$ können.

Anknüpfend an diesen Vorschlag bezogen wir die Kritik bereits in den Interpretationsprozess ein und unterzogen die objektiven Bedeutungsstrukturen $^{28}$ einer rassismuskritischen Reflexion. Die objektiven Bedeutungsstrukturen sind von rassistischem Alltagswissen durchzogen, hegemoniale Diskurse beziehen sich auf antimuslimische Stereotype als unhinterfragte Wissensbestände. ${ }^{29}$ Unseren Gesprächspartner:innen sind diese hegemonialen Diskurse bekannt, ohne dass sich ihr Handeln ausschließlich daran orientiert. Ihre Praxen, Perspektiven und Wissensbestände gehen in den hegemonialen Diskursen nicht auf, sie werden in der Regel nicht gesehen und gehört, sondern missrepräsentiert und missgedeutet, sodass es dazu kein Regelwissen

27 Leanza 2008: 103.

28 Objektive Bedeutungsstrukturen meint in der Methodologie der Objektiven Hermeneutik, dass jedes Mitglied einer Gesellschaft für seine Handlungen auf bestehende Strukturen und Regeln, die bereits bestehen, zurückgreift. Diese Strukturen und Regeln eignet sich der Mensch im Laufe seiner Sozialisation an. Das Ziel der Rekonstruktion liegt darin, diese herauszuarbeiten, sowie die Bedeutung der Auswahl von Handlungsmöglichkeiten zu verstehen (Wernet 2009: 13ff.).

29 Attia 2009: 53-94. 
gibt. Um sprechen zu können bzw. um gehört zu werden, orientieren sich unsere Gesprächspartner:innen bis zu einem gewissen Grad trotzdem an Themen, Begriffen, Metaphern und Sprachmotiven aus dem hegemonialen Islamdiskurs. Auf diese Weise können sie sich zunächst verständlich machen. Dies gilt auch für das Gespräch unter (als) Muslim:innen (Markierten), da sie miteinander bisweilen auch auf der Grundlage des hegemonialen Diskurses sprechen und dieser ihre geteilte Wirklichkeit (mit-)konstruiert. Aufgrund der rassismuskritischen Ausrichtung unserer Studie und der rassismuskritisch und intersektional informierten Interpretationsgruppen war es uns möglich, das Erfahrungswissen unserer rassifizierten Gesprächspartner:innen zu erkennen und die latenten Sinnstrukturen und Bedeutungen ihrer Lebenspraxen zu rekonstruieren. Insofern war es uns möglich, auch jene Strukturen, Erfahrungen und Wissensbestände zum Vorschein zu bringen, die nicht durch den Text und die ausgesprochenen Worte repräsentiert werden. Vielmehr erhielt das Gesagte vor dem Hintergrund von rassismusbezogenem Wissen, Theorien und Reflexionen seine Bedeutung.

Insofern konnten wir »die Selbstauffassungen des Handlungssubjekts, die Handlungsintentionen $\aleph^{30}$ vor dem Hintergrund des antimuslimischen Rassismus herausarbeiten. Die methodischen Schritte ${ }^{31}$ ließen sich problemlos anwenden und gewährleisten, dass die Lebenspraxis in ihrer Struktur rekonstruiert werden kann. Hierdurch lassen sich mehrere Umgangsstrategien und Subjektivierungsweisen herauskristallisieren. Wir fassten Gemeinsamkeiten mehrerer Einzelinterviews zusammen und stellen sie als Fallstruktur-Generalisierungen vor. Darunter versteht Wernet, dass

30 Wernet 2009: 18.

31 Oevermann versteht die Interpretation mithilfe der Objektiven Hermeneutik, als deren Begründer er gilt, als »Kunstlehre« (Oevermann 1993: 126) und gibt für die praktische Anwendung keine expliziten Vorgaben. Wernet hingegen hat in seinem Standardwerk Einführung in die Interpretationstechnik der Objektiven Hermeneutik»methodentechnische [...] Kernoperationen «(Wernet 2009: 10) für die Interpretation vorgestellt und diese anhand eines Lehrer:inneninterviews praktisch angewendet (ebd.: $52 \mathrm{ff}$.). 
»[d]er analysierte Fall [...] immer schon allgemein und besonders zugleich [ist]. Denn in jedem Protokoll sozialer Wirklichkeit ist das Allgemeine ebenso mitprotokolliert wie das Besondere im Sinne der Besonderheit des Falls. Der konkrete Fall ist insofern schon mehr als ein Einzelfall, als er ein sinnstrukturiertes Cebilde darstellt $«{ }^{32}$

In unserer Studie verstehen wir unter einem Fall Deutungen und Strategien. Folglich stellen wir die Fallstrukturgeneralisierungen jedes Einzelinterviews nicht einzeln vor, sondern fassen sie thematisch zusammen und stellen daher unterschiedliche Umgangsweisen mit dem Bedrohungsszenario vor.

\section{Schlussfolgerung}

Vor dem Hintergrund unserer Erfahrungen mit den genutzten rekonstruktiven Forschungsmethoden erscheint ihre macht- und rassismuskritische Anpassung geboten, sobald die Erfahrungen und Wissensbestände rassifizierter Personen interpretiert, analysiert und rekonstruiert werden. Wir halten dies auch für Forschungsfragen für angebracht, die nicht auf Rassismuserfahrungen fokussieren, da diese immer mitlaufen und relevant sein könnten. Bereits durch den groben Umriss unseres Forschungsinteresses und die Auswahl der Teilnehmer:innen an der Gruppendiskussion, deren Wissen um ihre eigenen Expertisen, denen der anderen und auch um unsere Expertisen in einem spezifischen Fachgebiet, geben wir einen bestimmten Rahmen vor. Diesen versuchen wir nicht zu kaschieren, indem wir etwa die Adressierung von (als) Muslim:innen (Markierten) zu vermeiden versuchen oder das Thema unserer Forschung vage halten. Vielmehr sprechen wir die Teilnehmer:innen explizit auf genau jene Adressierung an und rufen sie als Expert:innen ihrer selbst auf, halten uns aber in der Gruppendiskussion mit eigenen Beiträgen zurück und verbleiben in der Moderationsrolle. 
Uns interessieren sowohl explizite Äußerungen unserer Diskussionsteilnehmer:innen angesichts ihrer Erfahrung mit dem antimuslimischen Rassismus (kommunikatives Wissen) als auch jene handlungsleitenden, kollektiven (widerständigen) Wissensbestände, die sich konjunktiv artikulieren und der indirekten, gouvernementalen Selbstführung in Form alltäglicher Handlungen Rechnung tragen. Es geht uns also darum, die Wissensbegriffe in Dialog zu setzen, anstatt sie scharf voneinander zu trennen. Die Dokumentarische Methode schlägt dafür das Konzept des Orientierungsrahmens vor und verweist auf das Spannungsverhältnis beider Wissensformen. Sie geht davon aus, dass nur jene Normen, die im Kontrast zum eigenen Handeln erkannt werden, explizit formuliert werden können und deshalb kommunikativ sind, denn sonst würde die Norm in den Habitus übergehen und sich konjunktiv manifestieren. Nur jene Normen, die von Subjekten aktiv wahrgenommen werden, finden dementsprechend Eingang ins kommunikative Wissen, während sich im atheoretischen Wissen die impliziten, handlungsleitenden Wissensbestände befinden, da diese im konjunktiven Erfahrungsraum entstehen.

Vor dem Hintergrund intersektionaler, rassismuskritischer Theorien verstehen wir das üblicherweise als kommunikatives Wissen Bezeichnete als hegemoniales Wissen und meinen hiermit jenes öffentliche Wissen, das allen Gesellschaftsmitgliedern und damit auch Muslim:innen bekannt ist und im Grunde das situiert-kommunikative Wissen der Dominanzgesellschaft darstellt. Mit Erfahrungswissen oder situiertem Wissen meinen wir jenes kommunikativ-situierte Wissen unserer Diskussionsteilnehmer:innen, das ihnen darüberhinaus aufgrund ihrer Muslimisierung bekannt ist. Diese Spezifizierung eröffnet uns erst die Möglichkeit, unterschiedliche konjunktive Erfahrungsräume und damit unterschiedliche Umgangsweisen mit dem Sicherheitsdiskurs herauszuarbeiten. Sie basieren auf der Erfahrung des antimuslimischen Rassismus und dem Umgang mit dem Bedrohungsszenario und stellen damit die soziale Wirklichkeit von (als) Muslim:innen (Markierten) in einer Gesellschaft dar, die durch Rassismus strukturiert ist. 
